\title{
Somatosensory evoked potentials following stimulation of the lower limb in cortical reflex myoclonus
}

\author{
RYUSUKE KAKIGI, HIROSHI SHIBASAKI \\ From the Department of Internal Medicine (Division of Neurology), Saga Medical School, Nabeshima, Saga \\ City, Japan
}

SUMMARY Generating mechanisms of giant somatosensory evoked potentials (SEPs) following stimulation of the posterior tibial nerve as well as the big toe were investigated in three patients with cortical reflex myoclonus. Scalp distributions of recognisable components were very similar to those in normal subjects, except that their amplitude was much larger. The tibial nerve SEPs were remarkably attenuated by interfering tactile stimulation. Therefore, the giant SEPs observed in the present cases seem to be, at least partially, due to input from cutaneous nerve fibres on the background of extremely enhanced excitability in area $3 \mathrm{~b}$ of the primary sensory cortex where normal SEPs are generated

Somatosensory evoked potentials (SEPs) in patients with "cortical reflex myoclonus" 1 or "pyramidal myoclonus"2 are of remarkably high amplitude, and hence they have been commonly called "giant SEPs". Although there have been a number of reports concerning the giant SEPs following stimulation of the upper limbs, ${ }^{3-17}$ no systematic study of SEPs following stimulation of the lower limbs in cortical reflex myoclonus has been reported.

The present study, therefore, aimed at elucidating the pathophysiology of SEPs following stimulation of the lower limbs in cortical reflex myoclonus using various methods such as conventional nerve trunk stimulation, electrical finger stimulation, and the interference approach. ${ }^{18-22}$ In particular, the detailed scalp topography of each component was investigated.

\section{Patients and methods}

Three patients were carefully selected from those who manifested myoclonus, according to the following criteria: (1) the amplitude of the initial cortical positive potential recorded at or around the somatosensory leg area of the scalp following stimulation of the posterior tibial nerve at

Address for reprint requests: R Kakigi, MD, Department of Internal Medicine, Saga Medical School, Nabeshima, Saga City, 840-01, Japan.

Received 3 March 1987. Accepted 22 May 1987 the ankle was larger than the mean plus 2 standard deviations of normal subjects, (2) enhanced long-loop reflex (C reflex, termed by Sutton and Mayer $^{7}$ ) could be recorded even at rest (without voluntary contraction of the corresponding muscle), and (3) the presence of cortical spike was demonstrated by the jerk-locked averaging method ${ }^{91423}$ to precede a spontaneous myoclonic jerk.

All patients manifested intention as well as postural myoclonus, and they were taking normal doses of antimyoclonus medication, as listed in table 1 , to suppress myoclonus at the time of study. SEPs following stimulation of the upper limbs in all three cases were also of remarkably high amplitude.

Recordings were performed in a quiet, dimly lit room. The patient was kept awake in a reclining arm-chair, and was encouraged to relax. The electrical stimulus was a constant voltage square-wave pulse of $0.2 \mathrm{~ms}$ duration. It was delivered to the right posterior tibial nerve at the ankle. The stimulus intensity was sufficient to produce a definite twitch of the big toe. The right big toe was stimulated using silver ring electrodes with the stimulus intensity approximately $15 \mathrm{~mA}$.

Interfering tactile stimulus was applied by the experimenter by gently rubbing the right sole using a soft wad of tissue paper continuously and concurrently with the electrical stimulation of the posterior tibial nerve.

Silver disc recording electrodes were attached at 19 scalp locations based on the international 10-20 system. LFC, MFC, RFC, LCP, MCP and RCP electrodes were midway between corresponding sites of the 10-20 system as indicated in fig 1. All sites were referred to the linked earlobes (A1A2). The frequency response of the amplifiers was between 1 and $1500 \mathrm{~Hz}(-3 \mathrm{~dB})$. Five hundred responses to the posterior tibial nerve stimulation and 1000 responses to the big toe stimulation were averaged. At least three recordings were 
Table 1 Patients with cortical reflex myoclonus subjected to the present study

\begin{tabular}{lll}
\hline Case & $I$ & 2 \\
\hline Diagnosis & Progressive myoclonic epilepsy & Sialidosis type 2 \\
Sex and age & Female, 17 yr & Male, 55 yr \\
Other symptoms & Cerebellar ataxia & Cerebellar ataxia \\
Medication at the time of study & Clonazepam $6 \mathrm{mg}$ & Clonazepam $4 \mathrm{mg}$ \\
& Valproate $800 \mathrm{mg}$ & Valproate $600 \mathrm{mg}$ \\
& Primidone $500 \mathrm{mg}$ & Propranolol $60 \mathrm{mg}$
\end{tabular}

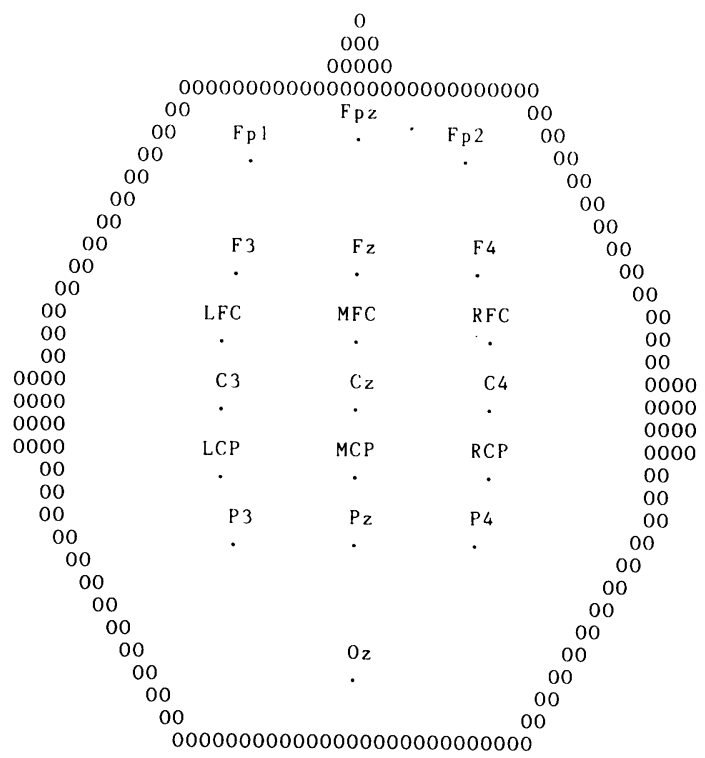

Fig 1 Electrode placements based on the international 10-20 system. LFC, MFC, RFC, LCP, MCP and RCP were midway between $F 3$ and $C 3, F z$ and $C z, F 4$ and $C 4$, $C 3$ and $P 3, C z$ and $P z$, and $C 4$ and $P 4$, respectively. The linked earlobes $(A 1+A 2)$ were used as the reference.

made for each stimulus condition. An analysis window was 5-125 ms for the posterior tibial nerve stimulation and $10-130 \mathrm{~ms}$ for the big toe stimulation. The sampling rate was $0.23 \mathrm{~ms}$. Responses contaminated with large artifact over $200 \mu \mathrm{V}$ were rejected by a computer program.
Each recognisable component was cursored for the peak latency and amplitude, the latter being measured from the preceding peak of the opposite polarity. The upper limit of the normal range was set to the mean plus 2 SD of the value obtained from 18 normal subjects in our department for each recording session (table 2).

Scalp topography of each consistent component was drawn using two different methods. In the first method, the topography was drawn using the peak-to-peak amplitude of each peak visually identified by the experimenter at each electrode to show its scalp distribution. The second method, which was the so-called isopotential map, was based on the amplitude from the baseline at each electrode at a particular time interval after the stimulus onset. The baseline was determined by averaging the deflection of the first $10 \mathrm{~ms}$ of the analysis window at each electrode.

Relative positivity at grid 1 resulted in a downward deflection in all recordings. Each recognisable component was named, because of the similarity of its wave form to thaț of the normal SEPs, according to the previous report of nor mal SEPs, ${ }^{21}$ and it was based on the polarity and peak latency.

\section{Results}

(1) SEPs following stimulation of the posterior tibial nerve at ankle

The wave forms recorded at the MCP and F3 electrode in the present cases as well as in one normal subject, whose data were selected as a representative in terms of the wave form and the scalp topography, are shown in fig 2. The amplitude of P39 and N48 at the MCP electrode was much larger than the normal range in all three cases (table 2 and fig 2). N33 was identified before P39 in all three cases, but its ampli-

Table 2 The amplitude of recognisable components in normal subjects as well as in patients with cortical reflex myoclonus in the present study

\begin{tabular}{|c|c|c|c|c|c|c|}
\hline \multirow[b]{3}{*}{ Stimulus site } & \multirow[b]{3}{*}{ Electrode } & \multirow[b]{3}{*}{ Component } & \multicolumn{4}{|l|}{ Amplitude $(\mu V)$} \\
\hline & & & \multirow{2}{*}{$\begin{array}{l}\text { Control }(n=18) \\
\text { mean } \pm S D\end{array}$} & \multicolumn{3}{|l|}{ Case } \\
\hline & & & & 1 & 2 & 3 \\
\hline $\begin{array}{l}\text { Posterior tibial nerve } \\
\text { at ankle }\end{array}$ & $\begin{array}{l}\text { MCP } \\
\text { F3 } \\
\text { MCP } \\
\text { F3 } \\
\text { MCP } \\
\text { F3 } \\
\text { MCP } \\
\text { F3 }\end{array}$ & $\begin{array}{l}\text { P39 } \\
\text { N39 } \\
\text { N48 } \\
\text { P48 } \\
\text { P39 } \\
\text { N39 } \\
\text { N48 } \\
\text { P48 }\end{array}$ & $\begin{array}{l}1.6 \pm 0.7 \\
1.0 \pm 0.4 \\
3.5 \pm 1.4 \\
1.5 \pm 0.7 \\
0.7 \pm 0.3 \\
0.3 \pm 0.2 \\
1.7 \pm 0.8 \\
0.7 \pm 0.3\end{array}$ & $\begin{array}{r}5 \cdot 1 \\
1.8 \\
15.6 \\
8.8 \\
4 \cdot 1 \\
1.0 \\
6.4 \\
2.5\end{array}$ & $\begin{array}{l}6.5 \\
2.7 \\
9.7 \\
5.0 \\
1.8 \\
1.0 \\
4.0 \\
1.6\end{array}$ & $\begin{array}{r}10 \cdot 0 \\
2.5 \\
10 \cdot 5 \\
6 \cdot 0 \\
2 \cdot 2 \\
0 \cdot 7 \\
5 \cdot 5 \\
2 \cdot 2\end{array}$ \\
\hline
\end{tabular}


Right posterior tibial nerve stimulation
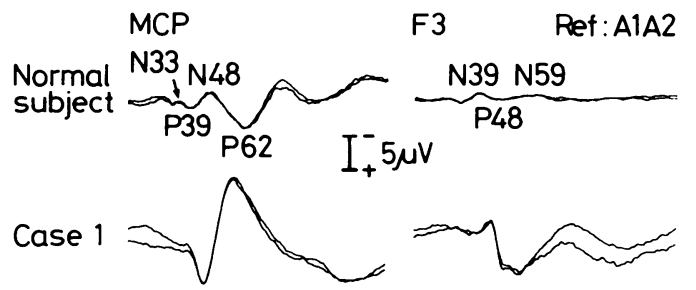

Case 2

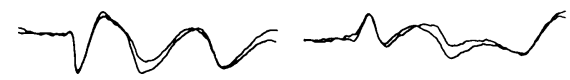

Case 3
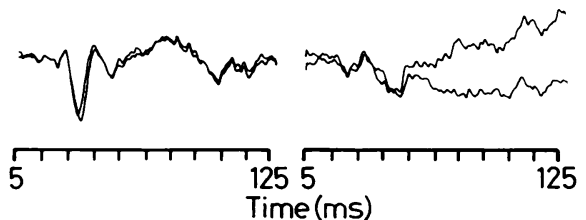

Fig 2 Wave forms of SEPs following stimulation of the right posterior tibial nerve at ankle recorded at two selected electrodes in one normal subject to show the nomenclature of each recognisable component, and in the present three cases. N33 is not so large in each case. In contrast, P39 and $N 48$ at the MCP as well as N39 and P48 at the F3 electrode are very large in the present cases.

Posterior tibial nerve stimulation Case 1

Ret: A1A2

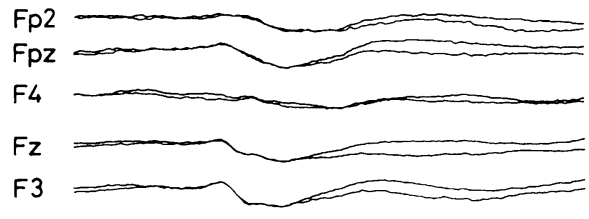

RFC

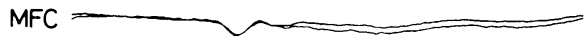

$\mathrm{LFC}$
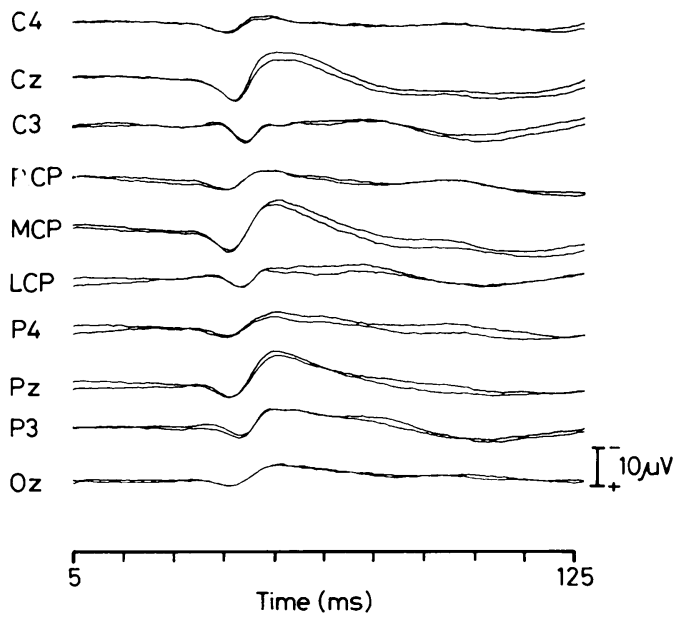

Fig 3 Wave forms of SEPs at all electrodes following stimulation of the right posterior tibial nerve at ankle in case 1.500 responses were averaged.
Right posterior tibial nerve stimulation

(a)

(b)

ref: $A 1 A 2$
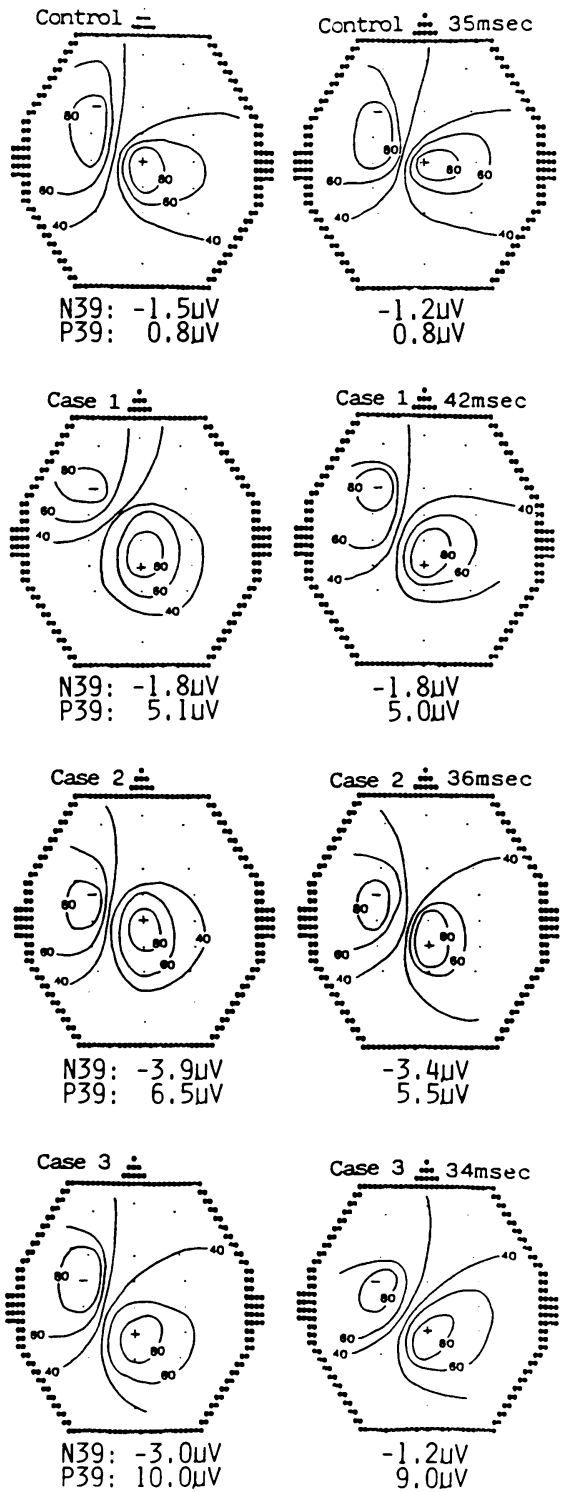

Fig 4 Scalp topography of P39-N39 complex in one normal subject as well as in each individual case. In (a), it was drawn using the peak-to-peak amplitude to show the distribution of P39 and N39, and in (b), it was drawn using the amplitude measured from the baseline at the latency when P39 was maximal in each individual record (isopotential maps). Electrode marked - or + shows the maximal point of the negative and positive component, respectively, and its amplitude is shown below each figure. 80,60 and 40 are the percentage amplitude with respect to the maximal point. $P 39$ is maximal at the $C z$ or $M C P$ electrode, and N39 is maximal at the F3 or LFC electrode in the normal subject as well as the present cases. 
Right posterior tibial nerve stimulation
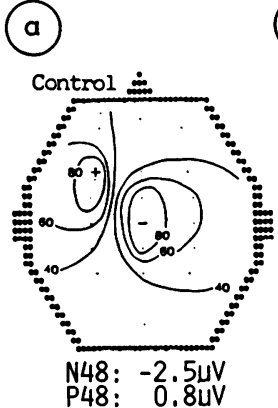

(b)

Ref : A1A2
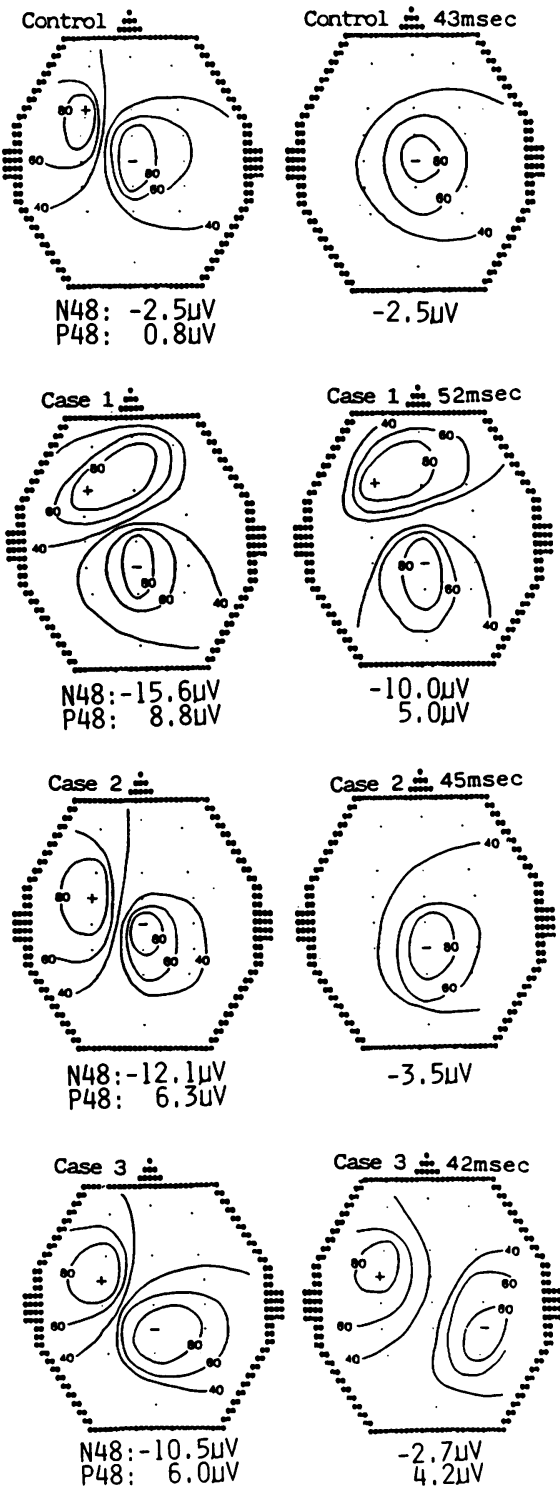

Fig 5 Scalp topography of N48-P48 complex based on the peak-to-peak amplitude (a), and isopotential maps at the latency when $N 48$ was maximal in each individual record (b). (refer to the legend for fig 4). In (a), N48 is maximal at the Cz or MCP electrode, and P48 is maximal at the F3 or LFC electrode in the normal subject as well as the present cases. However, the isopotential maps in (b) are relatively different from those in (a) probably because of the influence of the preceding P39 and N39 components. tude was within the normal range. The amplitude of N39 and P48 at the F3 electrode was also larger than the normal range, but was relatively smaller compared with P39 and N48 (table 2 and fig 2). The wave forms of components following N48 and P48 were variable from case to case. SEPs at all electrodes in case 1 were shown in fig 3 .

The peak latencies of P39, N39, N48 and P48 were within the normal range. The peak latencies of P39 and N48 at the MCP electrode showed no significant difference from those of $\mathrm{N} 39$ and P48 at the F3 electrode, respectively (paired $t$ test.).

Scalp distributions of P39-N39 and N48-P48 complexes in each case as well as in one normal subject were shown in figs $4 \mathrm{~A}$ and $5 \mathrm{~A}$, respectively. In all three cases, both $\mathrm{P} 39$ and N48 potentials were maximal at the MCP or $\mathrm{Cz}$ electrode, and spread in the central and parietal regions more to the hemisphere ipsilateral to the stimulated nerve. N39 and P48 were maximal at the F3 or LFC electrode, and spread to the frontal and central regions over the hemisphere contralateral to the stimulated nerve. Isopotential maps at the latency when P39 was maximal (fig 4b) were very similar to the scalp distribution of the P39-N39 complex (fig 4a), but those at the latency when N48 was maximal appeared different from the scalp distribution of N48-P48 complex in case 2 and

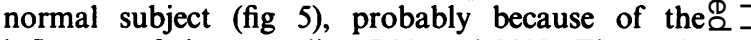
influence of the preceding P39 and N39. The scalp 0 distributions of those components in three cases were almost the same as those of our normal subjects aso well as those from the previous reports, ${ }^{21} 24$ in terms of the maximal point and distribution of each component, and of the orientation as well as the bordering area of their scalp distributions.

\section{(2) Interfering effects}

Interference effects by the continuous tactile stimulation applied to the sole ipsilateral to the stimulated nerve are shown at two selected electrodes, F3 and MCP (table 3 and fig 6). P39 and N48 at the MCP

Table 3 Amplitude changes of SEPs following stimulation of the right posterior tibial nerve at ankle due to concurrent interfering tactile stimulation of the right sole

\begin{tabular}{llllll}
\hline & \multicolumn{5}{c}{ Case } \\
\cline { 4 - 6 } Electrode & Component & Control* & $l$ & 2 & 3 \\
\hline MCP & P39 & $-34 \%$ & $-59 \%$ & $-27 \%$ & $-62 \%$ \\
F3 & N39 & $-21 \%$ & $-56 \%$ & $-46 \%$ & $-16 \%$ \\
MCP & N48 & $-33 \%$ & $-60 \%$ & $-29 \%$ & $-33 \%$ \\
F3 & P48 & $-27 \%$ & $-61 \%$ & $-47 \%$ & $-14 \%$ \\
F3 & N59 & $-10 \%$ & $-69 \%$ & $-20 \%$ & $+18 \%$ \\
MCP & P62 & $-15 \%$ & $-58 \%$ & $-3 \%$ & $-29 \%$ \\
\hline
\end{tabular}

*The average change due to interference in 18 normal subjects 
Right posterior tibial nerve stimulation

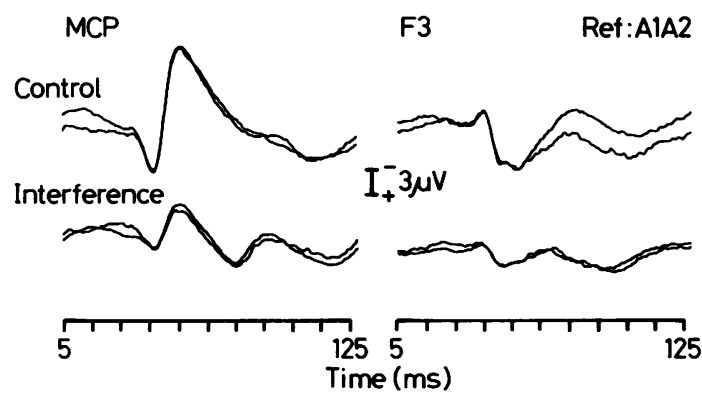

Fig 6 Wave form changes of SEPs at two selected electrodes following stimulation of the right posterior tibial nerve at ankle by concurrent tactile stimulation applied to the right sole in case 1. The upper traces are conventional SEPS (Control) and the lower ones are SEPS with interference (Interference). 500 responses were averaged. Each potential at each electrode is markedly attenuated by the tactile interference.

electrode were remarkably reduced in cases 1 and 3, and moderately reduced in case 2 . N39 and P48 at the F3 electrode were moderately reduced in cases 1 and 2 , and slightly reduced in case 3 . In contrast, the changes of the following components, N59 and P62, varied from subject to subject. Compared with the interference effects in normal subjects, the relative changes were consistently larger in case 1 , but were not different in cases 2 and 3 (table 3).

\section{(3) SEPs following stimulation of the big toe}

The wave forms of SEPs following electrical stimulation of the big toe by using ring electrodes (fig 7) were almost the same as those following the posterior

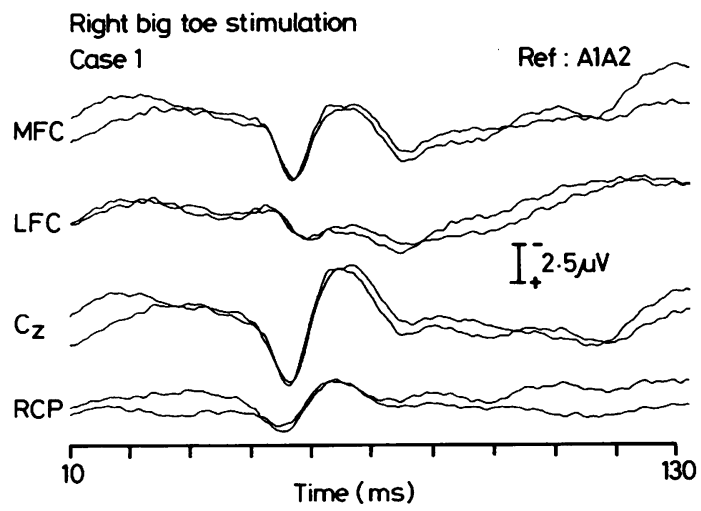

Fig 7 Wave forms of SEPs at four selected electrodes following stimulation of the right big toe in case 1.500 responses were averaged. The potentials, corresponding to P39, N39 and the following potentials to posterior tibial nerve stimulation, are also very large. tibial nerve stimulation. The amplitude was smaller than that to the posterior tibial nerve stimulation, but was much larger than the normal range in all cases (table 2). The scalp topographies of recognisable components were not significantly different from those of the corresponding components following stimulation of the posterior tibial nerve.

\section{Discussion}

To our knowledge, this is the first systematic study of SEPs following stimulation of the lower limb in cortical reflex myoclonus. P39, N39, N48 and P48 are considered to be cortical potentials like those in normal subjects, ${ }^{21}$ 24-30 since they showed a clearly localised distribution. Scalp distributions of P39 and N48 (figs 4 and 5) suggest that their generator source might be the leg region of the primary sensory cortex. Not only P39 and N48 recorded in the parietal region but also $\mathrm{N} 39$ and P48 recorded in the contralateral frontal region were larger than the normal range, and they were similarly reduced by tactile stimulation. Moreover, the difference of peak latencies between P39 and N39, or between N48 and P48 was not statistically significant. Therefore, it seems reasonable to consider that frontal potentials, N39 and P48, result from an orientation of dipolar activities generated in the primary sensory cortex, as reported in the studies of normal subjects. ${ }^{21252730}$

The components following stimulation of the big toe, corresponding to P39-N39 and N48-P48 of the tibial nerve SEPs, were also very large in each case, and their scalp distributions were very similar to those of the corresponding potentials following stimulation of the posterior tibial nerve. Moreover, the P39-N39 as well as N48-P48 complexes of the tibial nerve SEPs were considerably attenuated by tactile stimulation applied to the sole ipsilateral to the stimulated nerve in each case (fig 6 and table 3). These findings suggest that those potentials following the posterior tibial nerve stimulation might be, at least partially, due to input from cutaneous nerve fibres. This is compatible with the study of normal subjects in our previous report. $^{21}$ Therefore, the generator source for giant P39-N39 and N48-P48 complexes may contain, as for SEPs in normal subjects, ${ }^{21}$ area $3 b$ in the primary sensory cortex whose neurons receive input mainly from cutaneous nerve fibres. ${ }^{31}$

In conclusion, it seems reasonable to consider that giant SEPs following stimulation of the lower limb in cortical reflex myoclonus are generated by the same mechanisms as SEPs in normal subjects, although the excitability of the generator source, probably area $3 \mathrm{~b}$, must be extremely enhanced. This conclusion is not different from that for generating mechanisms of giant SEPs following stimulation of the upper limb in 
our previous report. ${ }^{17}$ In that study, ${ }^{17} \mathrm{~N} 19$ and P19, corresponding to the so-called $\overline{\mathrm{N}} 20$ and $\overline{\mathrm{P} 20}$, were not enlarged, but the following potentials, both $\overline{\mathbf{P} 25}$ in the contralateral parietal region and $\overline{\mathrm{N}} 25$ in the frontal region, were of remarkably large amplitude. Therefore, it seems reasonable to consider that the small negative deflection, N33 recorded at the parietal electrodes following the posterior tibial nerve stimulation, corresponds to $\overline{\mathrm{N}} 20$ of the median nerve SEPs, and that the following potentials, P39 and N39, correspond to $\overline{\mathrm{P} 25}$ and $\overline{\mathrm{N}} 25$ of the median nerve SEPs, respectively.

The authors are very grateful to Dr T Sakemi, Department of Internal Medicine, Saga Medical School, for allowing us to study case 3, and to Mrs J Matsuda for her technical assistance.

This study was supported by a grant from Myoclonus Research Fund, New Jersey, USA.

\section{References}

1 Hallett M, Chadwick D, Marsden CD. Cortical reflex myoclonus. Neurology 1979;29:1107-25.

2 Halliday AM. The electrophysiological study of myoclonus in man. Brain 1967;90:241-84.

3 Dawson GD. The relation between the electroencephalogram and muscle action potentials in certain convulsive states. $J$ Neurol Neurosurg Psychiatry 1946;9:5-22.

4 Dawson GD. Investigations on a patient subject to myoclonic seizures after sensory stimulation. $J$ Neurol Neurosurg Psychiatry 1947;10:141-62.

5 Watson CW, Denny-Brown D. Studies of the mechanism of stimulus-sensitive myoclonus in man. Electroencephalogr Clin Neurophysiol 1955;7:341-56.

6 Halliday AM, Halliday E. Cortical evoked potentials in patients with benign essential myoclonus and progressive myoclonic epilepsy. Electroencephalogr Clin Neurophysiol 1970;29:106-7.

7 Sutton GG, Mayer RF. Focal reflex myoclonus. J Neurol Neurosurg Psychiatry 1974;37:207-17.

8 Chadwick D, Hallett M, Harris R, Jenner P, Reynolds EH, Marsden CD. Cortical, biochemical, and physiological features distinguishing myoclonus responsive to 5-hydroxytryptophan, tryptophan with a monoamine oxidase inhibitor, and clonazepam. Brain 1977;100:455-87.

9 Shibasaki H, Yamashita Y, Kuroiwa Y. Electroencephalographic studies of myoclonus: myoclonus-related cortical spikes and high amplitude somatosensory evoked potentials. Brain 1978;101:447-60.

10 Kelly JJ Jr, Sharbrough FW, Daube JR. A clinical and electrophysiological evaluation of myoclonus. Neurology 1981;31:581-9.

11 Rothwell JC, Obeso JA, Marsden CD. On the significance of giant somatosensory evoked potentials in cortical myoclonus. J Neurol Neurosurg Psychiatry 1984;47:33-42.

12 Obeso JA, Rothwell JC, Marsden CD. The spectrum of cortical myoclonus. From focal reflex jerks to spontaneous motor epilepsy. Brain 1985;108:193-224.

13 Shibasaki H, Neshige R, Hashiba Y. Cortical excitability after myoclonus: jerk-locked somatosensory evoked potentials. Neurology 1985;35:36-41.
14 Shibasaki H, Yamashita Y, Neshige R, Tobimatsu S, Fukui R. Pathogenesis of giant somatosensory evoked potentials in progressive myoclonic epilepsy. Brain 1985;108:225-40.

15 Tobimatsu T, Fukui R, Shibasaki H, Kato M, Kuroiwa Y. Electrophysiological studies of myoclonus in sialidosis type 2. Elec troencephalogr Clin Neurophysiol 1985;60:16-22.

16 Cowan JMA, Rothwell JC, Wise RJS, Marsden CD. Electrophysiological and positron emission studies in a patient with cortical myoclonus, epilesia partialis continua and motor epilepsy. J Neurol Neurosurg Psychiatry 1986;49:796-807.

17 Kakigi R, Shibasaki H. Generator mechanisms of giant somatosensory evoked potentials in cortical reflex myoclonus. Brain (in press).

18 Jones SJ. An 'interference approach' to the study of somatosensory evoked potentials in man. Electroencephalogr Clin Neurophysiol 1981;52:517-30.

19 Jones SJ, Power CN. Scalp topography of human somatosensory evoked potentials: the effect of interfering tactile stimulation applied to the hand. Electroencephalogr Clin Neurophysiol 1984;58:25-36.

20 Kakigi R, Jones SJ. Effect on median nerve SEPs of tactile stimulation applied to adjacent and remote areas of the body surface. Electroencephalogr Clin Neurophysiol 1985;62:252-65.

21 Kakigi R, Jones SJ. Influence of concurrent tactile stimulation on somatosensory evoked potentials following posterior tibia nerve stimulation in man. Electroencephalogr Clin Neurophysiol 1986;65:118-29.

22 Kakigi R. Ipsilateral and contralateral SEP components following median nerve stimulation: effects of interfering stimuli applied to the contralateral hand. Electroencephalogr Clin Neurophysiol 1986;64:246-59.

23 Shibasaki H, Kuroiwa Y. Electroencephalographic correlates of myoclonus. Electroencephalogr Clin NeurophysiokD 1975;39:455-63.

24 Desmedt JE, Bourguet M. Color imaging of parietal and frontak somatosensory potentials fields evoked by stimulation of me- $O$ dian or posterior tibial nerve in man. Electroencephalogr Clin Neurophysiol 1985;62:1-17.

25 Cruse R, Klem G, Lesser RP, Lueders H. Paradoxical lat eralisation of cortical potentials evoked by stimulation of pos terior tibial nerve. Arch Neurol 1982;39:222-5.

26 Kakigi R, Shibasaki H, Hashizume A, Kuroiwa Y. Short latency somatosensory evoked spinal and scalp-recorded potentials following posterior tibial nerve stimulation in man. Electroencephalogr Clin Neurophysiol 1982;53:602-11.

27 Seyal M, Emerson RG, Pedley TA. Spinal and early scalprecorded components of somatosensory evoked potentials following stimulation of the posterior tibial nerve. Electroencephalogr Clin Neurophysiol 1983;55:320-30.

28 Kakigi R, Shibasaki H. Scalp topography of the short latency somatosensory evoked potentials following posterior tibia nerve stimulation in man. Electroencephalogr Clin Neurophysiol 1983;56:430-7.

29 Kakigi R, Shibasaki H, Hashizume A, Shima F, Kuroiwa Y. Short latency somatosensory evoked spinal, thalamic and scalp-recorded potentials following posterior tibial nerve stimulation in man. In: Homma S, Tamaki T, ed. Fundamentals and Clinical Application of Spinal Cord Monitoring. Tokyo: Saikon Press, 1984:345-60.

30 Kakigi R, Shibasaki $H$. The effect of aging on somatosensory evoked potentials following stimulation of the posterior tibia nerve in man. Electroencephalogr Clin Neurophysio 1987;68:277-86.

31 Paul RL, Merzenich M, Goodman H. Representation of slowly and rapidly adapting cutaneous mechanoreceptors of the hand in Brodmann's area 3 and 1 of Macaca mulatta. Brain Res 1972;36:229-49. 